\title{
Severe Asthma Patients Experience and Satisfaction with Virtual Clinics during COVID-19 Period
}

\author{
${ }^{1}$ College of Medicine King Saud University for Health Sciences \\ Riyadh Saudi Arabia \\ ${ }^{2}$ King Abdullah International Medical Research Centre (KAIMRC), \\ Riyadh, Saudi Arabia \\ 3 Pulmonary Division, Department of Medicine, Ministry of National \\ Guard Health Affairs, Riyadh, Saudi Arabia \\ ${ }^{4}$ Internal Medicine Division, Department of Medicine, Ministry of \\ National Guard Health Affairs, Riyadh, Saudi Arabia
}

Mohammad Ayaz Khan ${ }^{1,2,3}$ Rajendram Rajkumar ${ }^{1,2,4} \quad$ Mohammad Hammadi ${ }^{3}$

Majed Al-Gamedi ${ }^{1,2,3}$ Abdullah Al-Harbi ${ }^{1,2,3}$ Hamdan Al-Jahdali, ${ }^{1,2,3}$

Address for correspondence Hamdan Al-Jahdali, MD, FRCPC, FCCP, Pulmonary Division, Sleep Disorders Center, King Saud University for Health Sciences, King Abdulaziz Medical City, Riyadh, Saudi Arabia (e-mail: Jahdalih@gmail.com).

Avicenna J. Med. 2021;3:126-131.

\author{
Abstract \\ Keywords \\ - asthma \\ - biological therapy \\ - COVID-19 \\ - SARS-CoV-2 \\ - outpatient
}

Background Enforced social distancing (i.e., lockdowns) greatly facilitated control of coronavirus disease-19 (COVID-19). While access to hospitals was restricted, outpatient care continued remotely. The aim of this study was to determine the satisfaction of patients with severe asthma with telemedicine, and the impact of COVID-19 lockdown on severe asthma patients on biologics therapy.

Methods A cross-sectional survey of patients with severe asthma scheduled to receive biologic therapy at our hospital during the lockdown. The survey had sections about demographic data, asthma history, subjective perception of change in asthma control with biologic agent, the effect of COVID-19 pandemic on access to maintenance therapy for asthma, asthma exacerbation management, and satisfaction with telephone follow-up.

Results Fifty-four patients participated (response rate 93.1\%; male 17; mean age 46.7 years). All had been on biologic therapy for over 3 months (mean 38.4 months \pm standard deviation 26.5 months). Of the 45 patients living in Riyadh, 9 did not receive biologic therapy. Five of the nine patients living outside Riyadh did not receive biologic therapy. Alarmingly, 16 (29.6\%) had insufficient medications, and 27 (50\%) had difficulty obtaining medications. Fifty (92.6\%) had telephone follow-up, 31 (57.4\%) were satisfied with telemedicine.

Conclusion Many patients were satisfied with telemedicine, so this could be used to deliver routine outpatient tertiary care postpandemic. However, logistics around supplying medications and biologics must be considered in plans preparing for the second wave of COVID-19. published online August 4, 2021
DOI https://doi.org/

$10.1055 / \mathrm{s}-0041-1732283$

ISSN 2231-0770
(C) 2021 Syrian American Medical Society.

This is an open access article published by Thieme under the terms of the Creative Commons Attribution-NonDerivative-NonCommercial-License, permitting copying and reproduction so long as the original work is given appropriate credit. Contents may not be used for commercial purposes, or adapted, remixed, transformed or built upon. (https://creativecommons.org/licenses/by-nc-nd/4.0/).

Thieme Medical and Scientific Publishers Private Limited A-12, Second Floor, Sector -2, Noida-201301, Uttar Pradesh 


\section{Introduction}

During the coronavirus disease-19 (COVID-19) pandemic several research groups reported that fewer patients with asthma were admitted to hospital than expected..$^{1-3}$ This implied that patients with asthma were not at increased risk of complications from severe acute respiratory syndrome coronavirus 2 (SARS-CoV-2) infection..$^{1-3}$ However, more recent data from the United Kingdom suggests that patients with asthma, and particularly those with severe asthma, are at higher risk of in-hospital mortality from COVID-19. ${ }^{4}$

Enforced social distancing measures unintentionally created several barriers to the provision of health care for patients with chronic diseases. Curfews, imposed quarantines, and self-isolation were compounded by providers' fears of acquiring infection from patients, and patients' fears of acquiring infection from health care facilities. So, the Saudi Ministry of Health implemented several initiatives to engage the general public (e.g., smartphone applications and screening clinics) and advises health care facilities on how to maintain services while controlling the spread of SARS-CoV-2. ${ }^{5}$

Telehealth (i.e., virtual consultation) eliminates direct physical contact between patients and health care professionals during clinical encounters. Telehealth has many potential benefits especially for the routine follow-up of patients with known diagnoses. Thus, in view of the need to continue to provide outpatient care during the COVID-19 pandemic, telehealth gained popularity worldwide. ${ }^{6-8}$

Serendipitously, updated Saudi regulations on telemedicine were published by the National Health Information Centre in June 2019. These regulations laid the foundation for the rapid implementation of teleconsultations across the Kingdom during the COVID-19 pandemic. ${ }^{9}$ Health care providers, worldwide, reconfigured their practices, almost overnight. Telehealth was deployed in various forms including video calling, Web-based media, and telephone calls, to reduce the spread of COVID- 19.

Yet, in the 21st century, unenhanced telephone calls remain the most common medium for telehealth worldwide. The absence of visual cues makes assessments challenging. Moreover, objective measurements (e.g., peak expiratory flow, oxygen saturation) are not possible unless the patient has their own equipment. Regardless, the public seems to have been satisfied with the use of this technology to deliver health care during the COVID-19 pandemic. ${ }^{10}$

Select patients with severe asthma require inhaled steroid therapy and biologics therapy. There is currently no evidence to suggest that any medications for asthma increase the risk of SARS-CoV-2 infection or the severity of COVID-19. As loss of control of asthma can result in life-threatening complications, continuation of these medications is strongly recommended. ${ }^{11}$ Thus, patients with asthma must have sufficient supplies of their medications. To facilitate this hospital dispensaries and pharmacies must maintain adequate stocks. However, during the COVID-19 pandemic this was hindered by frequent disruptions of national and international supply chains.
Telemedicine initiatives have limited person-to-person contact and thus helped to reduce the spread of SARS-CoV-2. However, some patients may not have been able to continue their regular medications. The aim of this study was to determine the satisfaction of patients with severe asthma with virtual consultation and to evaluate the effect of the lockdown on patients' access to maintenance therapy for asthma.

\section{Materials and Methods}

\section{Study Setting and Design}

A cross-sectional, questionnaire-based survey study of patients with severe asthma receiving biologic therapy was performed at King Abdulaziz Medical city (KAMC), Riyadh, Saudi Arabia. This is a 1,600-bed academic tertiary care hospital. Biologic therapy is only prescribed after careful evaluation by a pulmonologist specializing in severe asthma.

During the early phase of COVID-19 pandemic, the KAMC established a protocol on telemedicine to transform the traditional outpatient practice to virtual clinics with a comprehensive framework for all clinical staff (Appendix 1). The physician in charge of the clinic contacted their patients by telephone. After their assessment, the physician will then agree a management plan with the patient and/or their relatives. For most patients this will include medical advice, ensuring that that patient has sufficient medications by prescribing any new or repeat medications as required, and arranging follow-up.

Patient satisfaction with telemedicine and the effects of the lockdown on access to maintenance therapy were investigated in a cohort of asthmatics on biologic therapy who attended virtual clinics from March 2020 until June 2020 (i.e., a 12-week period). The study cohort included all patients with severe asthma prescribed biologic therapy as add-on therapy who were under specialist care at KAMC. This cohort was chosen because each individual had a well-established diagnosis of asthma, expected to more symptoms, exacerbations, and had been managed using a traditional model of pulmonology outpatient care.

\section{Survey Components}

The survey had five sections. The first section collected standard demographic data (age, sex, education, ethnicity, employment, city of residence, attendance at other hospitals). The second investigated asthma history and use of biologic therapy (years since diagnosis of asthma, which biologic therapy is being used, duration of biologic therapy, and subjective perception of change in asthma control with biologic agent). The third section enquired about the effect of COVID-19 pandemic and social distancing on access to maintenance therapy for asthma (availability of medication, difficulty obtaining medications, missed scheduled biologic therapy during lockdown, and the reasons for this). The fourth explored satisfaction with telephone follow-up. The final section asked about the need for emergency treatment for asthma during the 
COVID-19 pandemic and the period of enforced social distancing (use of oral steroid for rescue, pharmacy visits, emergency department [ED] visits, and hospital admission).

\section{Study Outcomes}

Patients described their satisfaction with telemedicine and improvement with biologic therapy on a 5-point Likert scale (1 strongly disagree, 2 disagree, 3 neutral, 4 agree, 5 strongly agree). Patients' perception of medication sufficiency and the ease of accessing the hospital pharmacy were assessed on the same Likert scale. Closed questions (i.e., $\mathrm{Y} / \mathrm{N}$ ) were used to determine whether scheduled biologic therapy had been missed and if so the reasons for this were requested. Electronic medical records (EMRs) were also used to corroborate the subjects' answers. However, patients have access to other health care facilities besides KAMC. So, patients' accounts were used in preference to the EMR if there was any discrepancy.

\section{Statistical Analysis}

Statistical Product and Service Solutions (SPSS; version 20, IBM, United States) was used for data management and analysis. Categorical data were presented as frequency and percentage while interval data were described with mean and standard deviation (SD). The institutional review board (IRB) of the King Abdullah International Medical Research Center provided ethical approval for this study (IRBC.0686/20). Verbal informed consent was obtained from participants prior to their inclusion in the study.

\section{Results}

\section{Demographics}

The sample's demographic data are shown in - Table 1 . Fifty-four patients participated (female 37; male 17; mean age 46.7 years \pm SD 13.4 years). The response rate was excellent $(93.1 \%)$. However, some participants did not answer all of the questions. The majority of the sample were Saudi (51; 94.4\%), married (46; 85.2\%), resident in Riyadh (45; 83.3\%), and only received regular specialist follow-up for asthma at KAMC (44; 81.5\%). Four patients did not answer the question on marital status (3), educational achievement (4), and employment status (5).

\section{Duration of Asthma and Biological Therapy}

The mean time from diagnosis of asthma was 19.2 years (SD 11.5 years). All the patients in the sample had received biologic therapy for at least 2 months. Most patients were being treated with omalizumab $(45 ; 83.3 \%)$; the rest received mepolizumab $(7 ; 13.0 \%)$ and dupilumab (2; 3.7\%). Most of the sample agreed or strongly agreed that their symptoms of asthma had improved with biologic therapy (45; 83.3\%).

\section{Satisfaction with Telemedicine for Follow-Up of Asthma}

During the study period all participants received one telephone follow-up appointment in lieu of a scheduled in-person face-to-face appointment. However, not all patients were contactable during the time allocated for the virtual clinic. Of the 50 (92.6\%) patients who had telephone follow-up, 31 (57.4\%) were satisfied or very satisfied with this arrangement. However, five patients (9.2\%) were unsatisfied or very unsatisfied (-Table 2). When participants who were satisfied were compared with those who were not, no statistically significant differences in age, gender, employment status, level of education, marital status, failure to receive scheduled biologic therapy, difficulty obtaining medications, oral steroid use, pharmacy treatment, or ED visits, were found.

\section{Access to Maintenance Therapy for Asthma during the Lockdown}

At face-to-face follow-up appointments, medications prescribed in the clinic are usually dispensed from the hospital pharmacy on the same day. While medications were still prescribed at telephone follow-up, 17 participants (31.5\%) reported that it had subsequently been difficult to obtain medications from the hospital pharmacy (- Table 2).

Alarmingly, despite being "seen" in the telephonic virtual clinics, $16(29.7 \%)$ did not have sufficient medications to last until the next scheduled clinic visit ( - Table 2) and 14 (25.9\%) did not receive scheduled biologic therapy during the lockdown (-Table 3).

Table 1 Sample characteristics

\begin{tabular}{|c|c|c|}
\hline & & $n(\%)$ \\
\hline \multirow[t]{5}{*}{ Age (y) } & $22-30$ & $6(11.1)$ \\
\hline & $31-40$ & $15(27.8)$ \\
\hline & $41-50$ & $11(20.4)$ \\
\hline & $51-60$ & $13(24.1)$ \\
\hline & $61+$ & $9(16.7)$ \\
\hline \multirow[t]{2}{*}{ Gender } & Male & $17(31.5)$ \\
\hline & Female & $37(68.5)$ \\
\hline \multirow[t]{5}{*}{$\begin{array}{l}\text { Highest level of } \\
\text { educationa }\end{array}$} & $\begin{array}{l}\text { No formal } \\
\text { education }\end{array}$ & $8(14.8)$ \\
\hline & Elementary & $5(9.3)$ \\
\hline & Primary & $4(7.4)$ \\
\hline & High school & $11(20.3)$ \\
\hline & University & $22(40.7)$ \\
\hline \multirow{3}{*}{$\begin{array}{l}\text { Employment } \\
\text { status }^{\mathrm{a}}\end{array}$} & Employed & 21 (38.9) \\
\hline & Unemployed & $5(9.3)$ \\
\hline & Housewife & $23(42.6)$ \\
\hline \multirow[t]{4}{*}{ Marital status } & Single & $4(7.4)$ \\
\hline & Married & $46(85.2)$ \\
\hline & Widow & $3(5.6)$ \\
\hline & Divorced & $1(1.8)$ \\
\hline \multirow[t]{2}{*}{ City of residence } & Riyadh & $45(83.3)$ \\
\hline & $\begin{array}{l}\text { Outside } \\
\text { Riyadh }\end{array}$ & $9(16.7)$ \\
\hline
\end{tabular}

aSome answers were missing. 
Table 2 Patient satisfaction with telemedicine and access to maintenance therapy for asthma during the COVID-19 pandemic and lockdown

\begin{tabular}{|l|l|l|l|l|l|}
\hline Question & $\begin{array}{l}\text { Strongly } \\
\text { disagree }\end{array}$ & Disagree & Neutral & Agree & $\begin{array}{l}\text { Strongly } \\
\text { agree }\end{array}$ \\
\cline { 2 - 6 } & $n(\%)$ & $n(\%)$ & $n(\%)$ & $n(\%)$ & $n(\%)$ \\
\hline Sufficient medications until next clinic & $7(13.0)$ & $9(16.7)$ & $3(5.6)$ & $11(18.5)$ & $24(44.4)$ \\
\hline $\begin{array}{l}\text { Difficult to obtain medications from hos- } \\
\text { pital pharmacy }\end{array}$ & $21(38.9)$ & $9(16.7)$ & $7(13.0)$ & $10(18.5)$ & $7(13.0)$ \\
\hline $\begin{array}{l}\text { Satisfied with telephone follow up for } \\
\text { asthma }\end{array}$ & $2(3.7)$ & $3(5.6)$ & $18(33.3)$ & $14(25.9)$ & $17(31.5)$ \\
\hline Asthma improved with biologic therapy & $4(7.4)$ & $3(5.6)$ & $2(3.7)$ & $8(14.8)$ & $37(68.5)$ \\
\hline
\end{tabular}

\section{Scheduled Biologic Therapy during the Lockdown}

All patients in the sample were scheduled to receive biologic therapy during the lockdown. However, only 40 (74.1\%) received scheduled biologic therapy. Of the 14 patients who missed their biologic therapy, all bar one gave the reason for this on direct questioning (- Table 3). Of the 45 patients living in Riyadh, 9 did not receive biologic therapy, 2 cited the lockdown as the reason for this; two did not receive an appointment; two did not attend as they did not perceive any benefit; and two had other reasons. Five of the nine patients living outside Riyadh did not receive biologic therapy, three because of the lockdown, and one did not attend for fear of acquiring SARS-CoV-2.

\section{Self-Medication and Escalation of Asthma Therapy during the COVID-19 Pandemic and Lockdown}

Thirty-eight participants (70.4\%) did not need to escalate their treatment and none of the respondents were admitted to hospital during the COVID-19 pandemic and lockdown. However, as shown in - Table 4, 11 (20.4\%) decided to take over-the-counter treatment from a local pharmacy, 4 (7.4\%) took oral steroids, and 4 visited the ED. One respondent took over-the-counter treatment, started rescue therapy with oral steroids, and visited the ED; 5 patients used two of these three interventions and 10 required one. A total of 12 participants (22.2\%) self-medicated (i.e., took over-the-counter treatment, started oral steroids, or both).

\section{Discussion}

Enforced social distancing measures hampered the provision of health care to patients with chronic diseases during the COVID-19 pandemic. To overcome this, the ensconced,

Table 3 Reasons for missing biologic therapy during the lockdown

\begin{tabular}{|l|l|}
\hline Reason & $\boldsymbol{n}(\%)$ \\
\hline Curfew-related causes & $5(35.7)$ \\
\hline No improvement & $3(21.4)$ \\
\hline No appointment & $2(14.3)$ \\
\hline $\begin{array}{l}\text { Others (side effects, eligi- } \\
\text { bility, work) }\end{array}$ & $3(21.4)$ \\
\hline No reason given & $1(7.1)$ \\
\hline
\end{tabular}

Table 4 Patients who needed escalation of asthma therapy during the COVID-19 pandemic and lockdown

\begin{tabular}{|l|l|}
\hline & $N(\%)^{\mathrm{a}}$ \\
\hline $\begin{array}{l}\text { Emergency department visits after COVID-19 } \\
\text { lockdown }\end{array}$ & $4(7.4)$ \\
\hline $\begin{array}{l}\text { Additional for asthma from a local pharmacy/hospi- } \\
\text { tal during the lockdown }\end{array}$ & $11(20.4)$ \\
\hline Oral steroid use during the COVID-19 lockdown & $4(7.4)$ \\
\hline
\end{tabular}

${ }^{\mathrm{a}} \mathrm{As}$ explained in the text, some patients subjected to more than one interventions.

traditional, office-based outpatient model of the management of asthma had to be reconfigured rapidly. ${ }^{6}$ The spotlight was put on telemedicine and it rose to meet the challenge admirably.

We described the impact of telephone follow-up during the COVID-19 pandemic on a cohort of outpatients with severe asthma on biologic therapy being followed in a tertiary care center. The pulmonologists involved in these virtual clinics had to make difficult and complex decisions on whether to de-escalate therapy, continue current medications, or escalate to second- or third-line treatments.

Despite the limitations, over half of participants (57\%) were satisfied with using telemedicine that allowed the management of severe asthma during the lockdown. This is important to acknowledge because the basic model of telehealth described in the current study did not require any additional investment in terms of equipment, training, or staff time. Preexisting resources were utilized effectively. Furthermore, the use of telemedicine reduced the risk of harm to patients and staff while mitigating some of the growing risks associated with a steadily increasing backlog of outpatient work. However, approximately $10 \%$ of our sample was not satisfied with the simple model of telephone consultation described in the present study.

Previous studies have found that video consultations are associated with greater patient satisfaction than traditional office-based care, and do not negatively impact patients' outcomes. ${ }^{10,12}$ However, while studies and guidelines on telehealth consistently recommend high-tech solutions including online video consultations, ${ }^{9}$ these require increased resources in terms of cost, equipment, training, and staff time. The greater complexity also increases the risk of system failure. Furthermore, cultural issues must be considered. 
For example, a chaperone must be present when a female patient consults a male doctor in Saudi Arabia. While chaperones are also required for in-person consultations, they are not needed for telephone consultations.

Indeed, a simple telephone-based telehealth service was able to meet many of the needs of the patients in our sample. However, several logistical issues impeded the continuity of chronic health care during the lockdown. Although, reassuringly, our telephone survey generally found that our patients were in good health, a third of the sample reported that they did not have sufficient medications.

Although physicians had prescribed medications at telephone follow-up, a third of the sample had difficulty collecting medications from the hospital pharmacy. While a drive-through service was organized, patients with dwindling supplies were frequently prevented from collecting medication refills by curfews and pharmacy supply issues. Furthermore, while all participants were scheduled to receive biologic therapy during the lockdown, only $74 \%$ received this. Thus, continuing maintenance therapy for asthma during the lockdown was challenging.

The patients in this cohort had significant experience in the self-management of asthma (mean time from diagnosis was over 19 years). However, at least partly because of failure to receive regular maintenance medical therapy for asthma, $22 \%$ of the patients in this sample self-medicated with rescue oral steroids or took over-the-counter medications during the lockdown. While those who self-medicated during the lockdown will probably have used this strategy to avoid hospitalization prior to the pandemic, this observation highlights the severe consequences of the medication supply chain issues which occurred during the lockdown.

These logistical issues will be less relevant postpandemic, and moving forward, remote consultation has several advantages. Importantly, risks of cross-infection and disruption to patients' lives are minimized. Telehealth also promotes a shift from a hospital-centric, office-based chronic care to a more patient-centric model. However, to incorporate objective measurements into virtual asthma follow-up patients must be provided with monitoring equipment (e.g., peak expiratory flow meters and oxygen saturation probes).

Unfortunately, several sensitive legal issues remain unaddressed. For example, in many jurisdictions (including Saudi Arabia) it is unclear where the liability lies if a patient misunderstands instructions given during a teleconsultation. ${ }^{13,14}$ Ultimately, even video consultations cannot completely replace in-person face-to-face appointments. Indeed, there are several situations when teleconsultations are not suitable. As it is unlikely that health care facilities will be fully operational in the near future, these issues need to be considered when streaming patients to receive either remote or onsite consultations.

Furthermore, occasions will undoubtedly still arise when a patient's physical presence in clinic is unavoidable. ${ }^{15}$ However, in future the default should be teleconsultation, rather than physical presence at appointments on the hospital campus.

\section{Strengths and Limitations}

Self-reported data can be associated with response bias and so its accuracy can be questioned. However, participants' accounts of ED visits, prescribed medications, and attendance for administration of biologic therapy were consistent with hospital EMRs.

Studying a small, clearly defined cohort of patients with severe asthma receiving biologic therapy, attending a specialist clinic, at a single tertiary care center, provided good internal validity but limited the power of the study. Consequently, external validity and generalizability may be restricted. However, our survey had a high response rate (93\%), and our institution serves one of the largest caseloads of patients with severe asthma on biologics in Saudi Arabia.

The present study used a telephone model of telehealth. This is the most basic model of telemedicine for delivery of remote care. More advanced models of telemedicine incorporating online video-based technologies are available. However, in the 21st century, telephone follow-up remains the most commonly used model of telehealth worldwide. ${ }^{16}$ Our observations are therefore likely to be relevant to the follow-up of patients with severe asthma worldwide.

\section{Conclusion}

During the COVID-19 pandemic many patients with severe asthma were satisfied with the use of telemedicine for outpatient care. Patients were satisfied with the standard of the consultation and the prescription of their medications and home supplies. However, some patients did not receive scheduled biologic therapy during the lockdown. Thus, logistics around supplying medications and providing biologics must be considered to develop an effective and sustainable telemedicine model for outpatient care. For example, teaching patients to self-inject biologic therapy may facilitate this.

\section{Authors' Contributions}

H.J., M.K., and R.R. participated in the study concepts, design of the study, development of the questionnaire, and data acquisition and entry. M.H., M.G., and A.H. contributed in data analysis and statistical analysis of the data, participated in the intellectual content, reviewed and summarized the published literature and clinical studies, and participated in outlining the result themes and manuscript preparation, editing, and review. Corresponding author H.J. takes responsibility for the integrity of the work as a whole. All authors have critically reviewed and approved the final draft and are responsible for the content and similarity index of the manuscript.

\section{Funding}

This study was approved and funded by King Abdullah International Medical Research Center.

\section{Conflict of Interest}

All of the authors state that they have no conflicts of interest to declare. 


\section{Appendix 1: Protocol for telephone consultation during the COVID-19 pandemic}

- One day before the appointment a text message should be sent to prime patients to expect a telephone call from the hospital team the next day

- Greet and introduction

- Establish the identity of the person responding to the call

- Obtain verbal consent

- Obtain information on patient background clinical diagnoses

- Asked about current state of health including any exposure/disease with COVID-19

- Evaluate patients' activities of daily living and any change in symptoms

- Establish compliance with treatment including all inhalers, nebulizers, biologic injection treatment

- Review patient's medications and, if necessary, prescribe and arrange collection of a refill using pharmacy services' standard operating procedures

- Address any concerns of patients and family

- Following assessment decide whether the telephone consultation was adequate or in-person face-to-face evaluation is needed

- If the patient is stable advise them to stay at home. Alternatively, if the patient is unstable advise them to visit the hospital facility through special clinics or the Emergency Department (ED)

- Inform the patients/relatives of any investigations being requested

- Arrange a follow up appointment and bid the patient farewell

- Document the consultation in patient's electronic health record

\section{References}

1 Li X, Xu S, Yu M, et al. Risk factors for severity and mortality in adult COVID-19 inpatients in Wuhan. J Allergy Clin Immunol 2020;146(1):110-118

2 Zhang JJ, Dong X, Cao YY, et al. Clinical characteristics of 140 patients infected with SARS-CoV-2 in Wuhan, China. Allergy 2020;75(7):1730-1741

3 Lupia T, Scabini S, Mornese Pinna S, Di Perri G, De Rosa FG, Corcione S. 2019 novel coronavirus (2019-nCoV) outbreak: a new challenge. J Glob Antimicrob Resist 2020;21:22-27

4 Williamson EJ, Walker AJ, Bhaskaran K, et al. Factors associated with COVID-19-related death using OpenSAFELY. Nature 2020;584(7821):430-436

$5 \mathrm{MOH}$, Healthcare Transformation Strategy. Available at: https:// www.moh.gov.sa/en/Ministry/vro/Documents/HealthcareTransformation-Strategy.pdf. Accessed June 20, 2021

6 Hollander JE, Carr BG. Virtually perfect? Telemedicine for Covid-19. N Engl J Med 2020;382(18):1679-1681

7 HHS.gov. UDoHaHS. Notification of Enforcement Discretion for telehealth remote communications during the COVID-19 nationwide public health emergency. March 30, 2020. Available at: https://www.hhs.gov/hipaa/ for-professionals/special-topics/emergency-preparedness/ notification-enforcement-discretion-telehealth/index.html. Accessed June 10, 2021

8 Government. U. COVID-19: guidance for households with possible coronavirus infection. March 3, 2020. Available at: https://www.gov.uk/ government/publications/covid-19-stay-at-home-guidance/ stay-at-home-guidance-for-people-with-confirmed-or-possible-coronavirus-covid-19-infection. Accessed June 10, 2021

9 (SHC). NHICNiSHC. Telemedicine Regulations in the Kingdom of Saudi Arabia. June 29, 2018. Available at: https://nhic. gov.sa/en/Initiatives/Documents/Saudi\%20Arabia\%20 Telemedicine\%20Policy.pdf. Accessed June 10, 2021

10 Alghamdi SM, Alqahtani JS, Aldhahir AM. Current status of telehealth in Saudi Arabia during COVID-19. J Family Community Med 2020;27(3):208-211

11 Shaker MS, Oppenheimer J, Grayson M, et al. COVID-19: pandemic contingency planning for the allergy and immunology clinic. J Allergy Clin Immunol Pract 2020;8(5):1477-1488.e5

12 Armfield NR, Bradford M, Bradford NK. The clinical use of Skype-for which patients, with which problems and in which settings? A snapshot review of the literature. Int J Med Inform 2015;84(10):737-742

13 Alvandi M. Telemedicine and its role in revolutionizing healthcare delivery. Am J Accountable Care 2017;5(1):e1-e5

14 Alshammari F, Hassan S-u-N. Perceptions, preferences and experiences of telemedicine among users of Information and communication technology in Saudi Arabia. J Health Inform Dev Ctries 2019;13(1):1-13

15 Gupta A, Bush A, Nagakumar P. Asthma in children during the COVID-19 pandemic: lessons from lockdown and future directions for management. Lancet Respir Med 2020;8(11):1070-1071

16 Alaboudi A, Atkins A, Sharp B, Balkhair A, Alzahrani M, Sunbul T. Barriers and challenges in adopting Saudi telemedicine network: the perceptions of decision makers of healthcare facilities in Saudi Arabia. J Infect Public Health 2016;9(6):725-733 